\title{
Analytical Properties of Poincaré Halfmaps in a Class of Piecewise-Linear Dynamical Systems
}

\author{
Claus Kahlert and Otto E. Rössler \\ Institute for Physical and Theoretical Chemistry, University of Tübingen, Tübingen
}

Z. Naturforsch. 40 a, 1011-1025 (1985); received June 13, 1985

\begin{abstract}
A piecewise-linear nerve conduction equation is investigated further. The theory of Poincare halfmaps induced by the flow of a three-dimensional linear saddle-focus is developed. Using a description of the dynamical system in diagonalized coordinates, a canonical formulation of twodimensional halfmaps is found. This leads for each halfmap to an implicit scalar equation plus a side condition. The effect of the halfmaps on different types of invariant curves occurring is investigated. Thereby the capacity of the halfmaps to separate adjacent points (such that the images acquire a finite distance) is shown. Two of three possible mechanisms for separating points are investigated in detail. The regions in the canonical parameter space where the different separating mechanisms appear are indicated analytically. The possible appearance of chaotic solutions, at least in the neighborhood of homoclinic trajectories in state space, is demonstrated. The underlying separation mechanism is present also in regions of state space far from a homoclinic orbit.
\end{abstract}

\section{Introduction}

This is the first paper of a series treating the properties of piecewise-linear dynamical systems in terms of Poincaré halfmaps.

Piecewise-defined continuous dynamical systems are a very interesting class of systems because they allow to combine different types of dynamical behavior in different regions of state space. So this is the most general class possible. At first sight, requiring the partial dynamics that act on each of the regions to be linear, appears like a severe restriction. This is not the case, however. For a small enough size of the regions chosen, any nonlinear dynamical system can be approximated to any accuracy by a piecewise-linear one $[1,2]$. On the other hand, even those models using only a few regions in state space show a rich variety of types of qualitative dynamical behavior already (cf. [3, 4]). This aspect of piecewise-linear dynamical systems is more interesting than the capability to approximate arbitrary nonlinear systems. It seems as if most, if not all, types of qualitative dynamical behavior appear in "simple" (few-region) piecewise-linear systems. This class therefore provides rather easy to investigate prototypes for continuous nonlinear dynamical systems in general.

Reprint requests to Dr. C. Kahlert, Institut für Physikalische und Theoretische Chemie der Universität Tübingen, Auf der Morgenstelle 8, D-7400 Tübingen, F.R.G.
Piecewise-linear dynamical systems are especially amenable to analysis because inside each region of state space the solutions can be found in an elementary manner. The main problem is to determine the entry and exit points of a trajectory for each region. If the boundaries separating the regions are regular [5], the linear dynamics inside induce nonsingular point-transformations between the boundaries (or of one part of a boundary onto another part, respectively). For two-region dynamical systems these point-transformations are called Poincaré halfmaps $[6,7]$. This term stems from the fact that for these systems, the boundary between the regions - more precisely, that portion of the boundary where the trajectories cross from say region A to region $\mathrm{B}-$ may be used as a conventional Poincaré cross section. So a composition of the two "halfmaps" yields a Poincaré map.

The example treated here is among the simplest in three variables: It is the well-known RinzelKeller equation of nerve conduction [3], slightly modified $[2,8]$. The system contains just two regions separated by a flat plane, and the two dynamics differ only by the nonhomogeneous part of the right hand side. Unfortunately, the halfmaps that one meets in each region of state space can only be formulated implicitly. Nevertheless, some interesting substructures - which may be used to prove the possibility of chaotic solutions - can be calculated explicitly, using the analytical properties of halfmaps. 
The appearance of chaos in nerve conduction equations $[9,10]$ is one of the most surprising findings in the present class of systems. Nature divides the nerve fiber (or, as we will see, time) into roughly equidistant portions (cf. [8]), and then makes use only of the "pulse - nonpulse" (supra- or subthreshold) distinction as useful information, no matter what the detailed shape of the pulse. This means that the message transmitted by a nerve is completely encoded in the pulse sequence $[11,12]$. Chaotic solutions of the nerve conduction equation thus imply chaotic pulse sequences on the nerve. This, to us, points to the existence of a potentially rich "vocabulary".

\section{The Model System}

As our model system, we take a modified RinzelKeller equation [3] of nerve conduction [2,8],

$$
\begin{aligned}
& \partial_{t} u=D_{u u} \partial_{r}^{2} u+\mu[-u+v-b+\theta(u-d)], \\
& \partial_{t} v=-e u+v .
\end{aligned}
$$

Here the theta function is $\theta(x)=0$ if $x<0$ and $\theta(x)=1$ if $x>0$. (We leave the function undefined at $x=0$ for reasons of symmetry and will obtain trajectories at that point by a limiting process, cf. [5].) We rescale space by a factor of $1 / \sqrt{D_{u u}}$, introduce a wave variable $\varphi$ by looking for solutions of constant shape,

$$
\varphi:=r-c t,
$$

and take the first derivative of $u$ with respect to $\varphi$ as a new variable $w$. This leads us to a three-dimensional, first order ODE $[2,8]$,

$$
\begin{aligned}
\mathrm{d} u / \mathrm{d} \varphi & =w, \\
\mathrm{~d} v / \mathrm{d} \varphi & =(e u-v) / c, \\
\mathrm{~d} w / \mathrm{d} \varphi & =\mu[u-v+b-\theta(u-d)]-c w .
\end{aligned}
$$

For a detailed discussion of the procedure leading from (1) to (3) cf. [2]. Equation (3) is our basic equation of motion to be considered further on. Compared to the original reaction-diffusion-equation (1), it contains an additional "free parameter", the wave speed $c$ (a property of the wave-solution). This parameter has to be chosen in such a way as to fulfill the boundary conditions of the original PDE [2], leading to a nonlinear eigenvalue problem.
Let us first discuss the geometry of the state space of the dynamical system (3). The $\theta$ function in the nonhomogeneous part of (3) divides the state space $T$ into two halves,

$$
\bar{T}:=\left\{(u, v, w)^{T} \mid u<d\right\}
$$

and

$$
\tilde{T}:=\left\{(u, v, w)^{T} \mid u>d\right\},
$$

with the flat separating plane

$$
S:=\left\{(u, v, w)^{T} \mid u=d\right\} .
$$

in between. It is helpful to introduce

$$
\bar{\sigma}:=1 \text { and } \tilde{\sigma}:=-1,
$$

so that (4) now reads

$$
\hat{T}:=\left\{(u, v, w)^{T} \mid \hat{\sigma} u<\hat{\sigma} d\right\} .
$$

Here the "^" is either a " ", or a " " ", respectively.

The steady states $(\hat{L})$ of $(3)$ are easily found:

$$
\begin{aligned}
& \hat{U}=\frac{2 b-(1-\hat{\sigma})}{2(e-1)}, \\
& \hat{V}=e \hat{U}, \\
& \hat{W}=0 .
\end{aligned}
$$

Transforming (3) in such a way that the steady state $\bar{L}$ (or $\tilde{L}$, respectively) becomes the origin, both partial dynamics read

$$
\frac{\mathrm{d}}{\mathrm{d} \varphi}\left(\begin{array}{l}
u \\
v \\
w
\end{array}\right)=\left(\begin{array}{ccc}
0 & 0 & 1 \\
e / c & -1 / c & 0 \\
\mu & -\mu & c
\end{array}\right)\left(\begin{array}{l}
u \\
v \\
w
\end{array}\right)
$$

or equivalently

$$
\frac{\mathrm{d}}{\mathrm{d} \varphi} \boldsymbol{l}=\mathbb{B} \boldsymbol{l} .
$$

Here $\boldsymbol{l}$ is the state vector and $\mathbb{B}$ the dynamical matrix. Further on we shall always use steady state coordinates, such as $\bar{u}=u-\bar{U}$, for example. In addition we omit the " " " for the coordinates, as we already did in (8).

Only for parameter values

$$
e \cdot d>b+d,
$$

and

$$
e>1 \text {, }
$$

excitations (and hence wave propagation) phenomena appear [13]. So only this part of the parameter 
space of (1) needs to be considered. Here we find (from (9)) that

$$
d>b /(e-1)=\bar{U} .
$$

The steady state $\bar{L}$ thus is situated inside $\bar{T}$, i.e., it is a real steady state. On the other hand, we also obtain from (9)

$$
\tilde{U}=\frac{b-1}{e-1}<\frac{b}{e-1}<d .
$$

This means that the steady state $\tilde{L}$ too is situated inside $\bar{T}$. It is a virtual steady state [13] for the dynamics of $\tilde{T}$.

Some words on the dynamics of the model system (3). The spectrum of the matrix $\mathbb{B}$ as a function of the wave speed $c$ is shown and discussed in [2], so we skip this topic here and mention only two general aspects. First, the steady states are always unstable for $\mu>1$. The characteristic equation of $\mathbb{B}$ reads

$$
\lambda^{3}-\lambda^{2} \operatorname{tr} \mathbb{B}+\lambda \min \mathbb{B}-\operatorname{det} \mathbb{B}=0,
$$

whereby

$$
\begin{aligned}
& \operatorname{tr} \mathbb{B}=-\frac{c^{2}+1}{c}, \quad \min \mathbb{B}=1-\mu, \\
& \operatorname{det} \mathbb{B}=\frac{-\mu}{c}(e-1) .
\end{aligned}
$$

This cubic equation possesses (by Descartes' rule) always one real negative root $\left(\lambda_{1}\right)$ and two positive real or complex conjugate roots $\left(\lambda_{2}, \lambda_{3}\right)$. Restricting our attention to the latter case, we factorize (12) to obtain

$$
\begin{aligned}
0= & \left(\lambda-\lambda_{1}\right) \cdot\left(\lambda-\lambda_{2}\right) \cdot\left(\lambda-\lambda_{3}\right) \\
= & \lambda^{3}-\lambda^{2}\left(\lambda_{1}+2 \operatorname{Re} \lambda_{2}\right) \\
& +\lambda\left(2 \lambda_{1} \operatorname{Re} \lambda_{2}+\left|\lambda_{2}\right|^{2}\right)-\lambda_{1}\left|\lambda_{2}\right|^{2} .
\end{aligned}
$$

Comparing the coefficient of (12) and (13) for $\lambda$ leads one to

$$
\left|\lambda_{2}\right|^{2}+2 \lambda_{1} \operatorname{Re} \lambda_{2}=1-\mu .
$$

Thus

$$
\operatorname{Re} \lambda_{2}=\frac{1-\mu-\left|\lambda_{2}\right|^{2}}{2 \lambda_{1}}
$$

As $\mu>1$ and $\lambda_{1}<0, \operatorname{Re} \lambda_{2}$ will always be positive and hence the focal parts of the steady states are unstable.
Second, a certain quantitative relation between the eigenvalues is always fulfilled. Comparing the coefficients of $\lambda^{2}$ from (12) and (13) one finds

$$
-\left(\lambda_{1}+2 \operatorname{Re} \lambda_{2}\right)=\frac{c^{2}+1}{c},
$$

that is

$$
-\lambda_{1}=2 \operatorname{Re} \lambda_{2}+\left(c^{2}+1\right) / c .
$$

Hence

$$
-\lambda_{1}>2 \operatorname{Re} \lambda_{2},
$$

or

$$
\left|\lambda_{1}\right| / \operatorname{Re} \lambda_{2}>2 .
$$

The latter relation between the first eigenvalue and the real part of the second means that the system fulfills the prerequisites of Shil'nikov's theorem [14] $\left(\left|\lambda_{1}\right|>\operatorname{Re} \lambda_{2}\right)$, and therefore may possess at least one type of chaotic solutions if a homoclinic trajectory in state space is present (see Section 5).

As to the eigenvectors of the dynamical matrix $\mathbb{B}$, they are easily found to be

$$
t^{i}=\left(\begin{array}{c}
1 \\
e \\
1+i_{i} c \\
i_{i}
\end{array}\right),
$$

(cf. [2]), determining the transformation matrix

$$
\mathbb{H}:=\left(t^{1}, t^{2}, t^{3}\right)
$$

which diagonalizes $\mathbb{B}$ :

$$
\boldsymbol{\Lambda}:=\mathbb{H}^{-1} \mathbb{B H}=\left(\begin{array}{lll}
\lambda_{1} & 0 & 0 \\
0 & \lambda_{2} & 0 \\
0 & 0 & \lambda_{3}
\end{array}\right) .
$$

Thus the state vectors transform like

$$
\boldsymbol{l}=\left(\begin{array}{l}
u \\
v \\
w
\end{array}\right)=\mathbb{H}\left(\begin{array}{l}
x \\
y \\
z
\end{array}\right)=\mathbb{H} k,
$$

or equivalently

$$
k=\mathbb{H}^{-1} \boldsymbol{l} .
$$

The dynamics of system (8) in the diagonalized coordinates ( $k$-space, using the eigenvectors (16) as a basis) now simply reads

$$
\frac{\mathrm{d}}{\mathrm{d} \varphi} k=\Lambda k \text {. }
$$


Of particular interest are the transformation properties of the switching condition in the separating plane $S$, transformed to steady state coordinates. We obtain for this condition $(u=d-\hat{U}=: \hat{\delta})$, when written in diagonalized coordinates,

$$
x+y+z=x+2 \operatorname{Re} y=\delta .
$$

Putting

$$
\eta:=\operatorname{Re} y \quad \text { and } \quad \xi:=\operatorname{Im} y
$$

the switching condition (21) simplifies to

$$
x+2 \eta=\delta \text {. }
$$

The formulation of the dynamics in (20) describes the system in the state space $\mathbb{R} \times \mathbb{C}^{2}$. As two of the eigenvalues $\left(\lambda_{2}\right.$ and $\left.\lambda_{3}\right)$ are complex conjugate, necessarily a side condition $z=\bar{y}$ arises. It therefore appears more convenient to reformulate the dynamics of the system in the space $\mathbb{R} \times \mathbb{C}$ (in $x, y$ coordinates) without a side condition.

As (23) already fits into this representation, the separating plane $S$ is written now as

$$
S=\left\{(x, y)^{T} \mid \eta=\frac{\delta-x}{2}\right\} .
$$

This means that all points of $S$ are characterized uniquely by their $x$ and $\xi$ coordinates while $\eta$ becomes a function of $x$ alone. A reformulation of the dynamics in the new state space will be given in the next Section.

\section{The Canonical Parameter Space}

After having considered a concrete model system, let us now define a more abstract class of systems possessing the required properties. Firstly, the geometry of the state space is to be described by the position of the separating plane $S$ and the displacement of the two steady states from this plane. Hence the difference vector between the steady states $\left(\Delta=\left(\Delta_{1}, \Delta_{2}, \Delta_{3}\right)^{T}:=\bar{L}-\tilde{L}\right)$ and the position of $S$ are not completely mutually independent:

$$
\begin{aligned}
\bar{\delta}-\tilde{\delta} & =(d-\bar{U})-(d-\tilde{U}) \\
& =-\Delta_{1},
\end{aligned}
$$

and

$$
\Delta_{3}=\bar{W}-\tilde{W}=0 .
$$

As far as purely geometrical parameters are concerned, there remain only $\bar{\delta}, \tilde{\delta}$, and $\Delta_{2}$. In addition, there is one free parameter left: An arbitrary shift of the whole system in the $r$ direction. Since this causes only a parallel shift of equivalent halfmaps, it generates no new structures and can thus be ignored.

Let us now turn to the dynamical parameters. The wave variable $\varphi$ appears in (3) as a time-like quantity. As we are only interested in the Poincaré halfmaps induced by the flow of each partial system, we do not need to know the "speed" at which the system follows a trajectory. We therefore define a new "time", $\varphi^{\prime}$, as

$$
\varphi^{\prime}:=\varphi \operatorname{Re} \lambda_{2} .
$$

The derivatives with respect to this new variable transform like

$$
\frac{\mathrm{d}}{\mathrm{d} \varphi^{\prime}}=\frac{1}{\operatorname{Re} \lambda_{2}} \frac{\mathrm{d}}{\mathrm{d} \varphi} .
$$

Since the $w$ variable was already interpreted as the derivative of $u$ with respect to $\varphi$, it now has to be redefined:

$$
w^{\prime}:=\frac{\mathrm{d} u}{\mathrm{~d} \varphi^{\prime}}=\frac{w}{\operatorname{Re} i_{2}} .
$$

The use of $\varphi^{\prime}$ leads to a "normalization" of the speed at which the system runs along a trajectory.

For abbreviation, we introduce the canonical dynamical parameters:

$$
\varrho:=\frac{\left|\lambda_{1}\right|}{\operatorname{Re} \lambda_{2}}
$$

and

$$
\omega:=\left|\frac{\operatorname{Im} \lambda_{2}}{\operatorname{Re} \lambda_{2}}\right| .
$$

Hence for our equations of motion, we obtain in the old coordinates

$$
\begin{aligned}
\frac{\mathrm{d} u}{\mathrm{~d} \varphi^{\prime}} & =w^{\prime}, \\
\frac{\mathrm{d} v}{\mathrm{~d} \varphi^{\prime}} & =\frac{e u-v}{\operatorname{Re} \lambda_{2} c}, \\
\frac{\mathrm{d} w}{\mathrm{~d} \varphi^{\prime}} & =\frac{\mu}{\operatorname{Re} \lambda_{2}}[u-v+b-\theta(u-d)]-c w^{\prime}
\end{aligned}
$$

and in the new (diagonalized) coordinates:

$$
\begin{aligned}
& \mathrm{d} x / \mathrm{d} \varphi^{\prime}=-\varrho x, \\
& \mathrm{~d} y / \mathrm{d} \varphi^{\prime}=(1+i \omega) y .
\end{aligned}
$$


This shows that $\varrho$ is the relaxation constant in the direction of the real eigenvector ( $x$ direction) while $\omega$ is the angular velocity of the focal motion. From now on we omit the primes since only the new variables will be used.

As can be seen from (32), we have, by a (in parameter space local) gauging, condensed the information of the formerly three (complex) eigenvalues into two real numbers, namely, $\varrho$ and $\omega$. The class of systems to be considered therefore now contains five parameters $\left(\bar{\delta}, \tilde{\delta}, \Delta_{2}, \varrho, \omega\right)$ which completely classify the Poincare halfmaps. The remaining two degrees of freedom (a $v$ shift and an arbitrary nonsingular transformation on $\varphi$ ) do not affect the character of the halfmaps.

Let us condense the properties of interest of our class of systems into two axioms.

SY1: The systems possess one real and one virtual steady state.

This axiom reflects the properties of the model system (3). As will be shown, only one partial system (the one governed by the real steady state) needs to be considered in detail.

SY2: Both steady states are of the saddle-focus type and possess the same eigenvalues and eigenvectors.

This is the most relevant axiom because it restricts the type of dynamical behavior that gives rise to the halfmaps. (A third axiom "SY3" will be added for purely technical reasons in Section 5.)

\section{Formulation of the Halfmaps}

Inside each region of state space ( $\bar{T}$ or $\tilde{T}$, respectively) the trajectories of the system can be indicated explicitly. For initial condition, say $(\bar{\alpha}, \bar{\beta})^{T} \in \bar{T}$, the solution of (32) reads

$$
\begin{aligned}
x(\varphi) & =\bar{\alpha} \exp (-\varrho \varphi), \\
y(\varphi) & =\bar{\beta} \exp ((1+\mathrm{i} \omega) \varphi) .
\end{aligned}
$$

This solution is valid as long as $(x(\varphi), y(\varphi))^{T}$ is from $\bar{T}$. If at $\varphi=\tau$ the trajectory meets the requirements of the switching condition (23), the second dynamics takes over; here the initial condition is

$$
\begin{aligned}
& \tilde{\alpha}=x(\tau)+(\bar{X}-\tilde{X}), \\
& \tilde{\beta}=y(\tau)+(\bar{Y}-\tilde{Y}) .
\end{aligned}
$$

As the canonical parameters $\varrho$ and $\omega$ are identical for both partial systems, the functional shape of the trajectory in $\tilde{T}$ is again given by (33) (now with initial condition (34)). So if we know all the entry points (or the exit points, equivalently, situated inside $S$ ) of a trajectory, the solution is completely determined.

Owing to the two different possible orientations that the flow may have as it passes through $S$, this plane is divided in a natural manner into two halves:

$$
S^{+}:=\left\{(u, v, w)^{T} \mid u=\delta, w>0\right\}
$$

and

$$
S^{-}:=\left\{(u, v, w)^{T} \mid u=\delta, w<0\right\} .
$$

The simplicity of this division is due to the linearity of the flows. Inside $S^{+}$, the system always crosses the boundary from $\bar{T}$ towards $\tilde{T}$, while the opposite holds true for $S^{-}$. These two halfplanes are separated by a straight line,

$$
W:=\left\{(u, v, w)^{T} \mid u=\delta, w=0\right\} .
$$

Along this line the trajectories are tangential to $S$. In diagonalized $(x, \xi)$ coordinates $W$, may be written as

$$
\xi=\frac{\delta-(\varrho+1) x}{2 \omega} .
$$

This follows from (36) and the transformation equation (19).

We are now in the position to formulate the two Poincare halfmaps $\bar{P}$ and $\tilde{P}$ that are induced by the flows inside $\bar{T}$ and $\tilde{T}$, respectively:

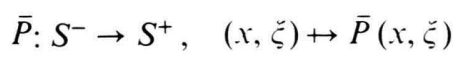

and

$$
\tilde{P}: S^{+} \rightarrow S^{-}, \quad(x, \xi) \mapsto \tilde{P}(x, \xi) .
$$

The complete Poincaré map $P$ is, as mentioned, a composition of these halfmaps:

$$
\begin{aligned}
P=\tilde{P} \circ \bar{P}: S^{-} & \rightarrow S^{-}, \\
(x, \xi) & \mapsto P(x, \xi)=\tilde{P}(\bar{P}(x, \xi)) .
\end{aligned}
$$

By inserting the solution (33) into the switching condition (23), we obtain a concrete formulation of each halfmap:

$x e^{-\varrho \tau}+2 \operatorname{Re}[(\eta(x)+\mathrm{i} \xi) \exp \{(1+\mathrm{i} \omega) \tau\}]=\delta$.

This is an implicit scalar algebraic equation. Here $\eta(x)$ is nothing but a reformulation of the switching 
condition (23) - namely the condition that $(x, \eta(x)+\mathrm{i} \xi)^{T} \in S-$ written in the form

$$
\eta(x)=(\delta-x) / 2 .
$$

In addition, since ( $40 \mathrm{a})$ contains infinitely many solutions, we must select the one that is relevant for the halfmap by requiring

$$
\begin{gathered}
\hat{\sigma}\left(x e^{-\varrho \varphi}+2 \operatorname{Re}[(\eta(x)+\mathrm{i} \xi) \exp \{(1+\mathrm{i} \omega) \varphi\}]\right) \\
<\hat{\sigma} \delta, \quad 0<\varphi<\tau .
\end{gathered}
$$

This inequality guarantees that the solution $\tau$ of (40 a) is actually the first one possible in $\mathbb{R}^{+}$(the trajectory has stayed inside $\hat{T}$ for all positive values of $\varphi$ that are smaller than $\tau$ ). This condition will sometimes be omitted in the following in order then to be reintroduced as a selection rule (cf. Section 6).

The implicit equation (40a) with side condition (40 b) cannot be solved explicitly for all points of $S^{+}$ (or $S^{-}$, respectively). Nevertheless, it can be solved analytically for some singular points. Moreover the images of special curves in $S$ can be calculated (Sections 6 through 8).

\section{Statical and Dynamical Manifolds}

In the preceding Section it was shown how a trajectory is completely defined by all its entry and exit points of the two regions $\bar{T}$ and $\tilde{T}$, respectively. Hence the structures that are generated by these points, inside the separating plane $S$, determine the phase portrait of the dynamical system. On the other hand, the properties of structures in state space (like manifolds), can too be found again as properties of sets of points in $S$.

As the two partial dynamics are linear, the stable and unstable manifolds of the steady states coincide inside each halfspace with those spanned by their eigenvectors. Hence the two stable manifolds are simply

$$
\hat{M}_{\mathrm{s}}:=\hat{K}+\varkappa t^{2} . \quad \varkappa \in \mathbb{C}
$$

(with the " " "again standing for either ".-", or ". ", respectively). These are the planes of the two steady states (of saddle-focus type) which contain the foci. Their intersections with the separating plane $S$ are two straight lines,

$$
\hat{\Sigma}:=\left\{(x, y)^{T} \mid x=0, \quad \eta=\hat{\eta}_{\infty}\right\},
$$

whereby

$$
\hat{\eta}_{\infty}:=\eta(0)=\frac{\delta}{2}
$$

cf. (41).

The two parallel lines $\bar{\Sigma}$ and $\tilde{\Sigma}$ cut out a strip $S^{0}$ from $S$, cf. Figure 1. Within this strip $S^{0}$, almost all of the entry and exit points of any concrete solution will be found. This is due to the fact that every trajectory with finite initial condition necessarily relaxes in the direction towards the stable manifold that is pertinent and will therefore, sooner or later, enter the (unbounded) space between the two parallel planes $\bar{M}_{s}$ and $\tilde{M}_{s}$ in order to remain there forever. Specifically all periodic and nonperiodic limiting structures of the Poincaré map $P$ can be found inside of $S^{0}$.

This strip $S^{0}$ can (like $S$ itself) now be divided up further into the two portions

$$
S^{0+}:=S^{0} \cap S^{+}
$$

and

$$
S^{0-}:=S^{0} \cap S^{-} .
$$

Turning to the unstable manifolds, their equations

$$
\hat{M}_{u}:=\hat{K}+v t^{1}, \quad v \in \mathbb{R},
$$

are especially simple since they are spanned by the real eigenvector alone. The intersection points of these two manifolds with $S$ are

$$
\hat{h}:=\left\{(x, y)^{T} \mid x=\delta, y=0\right\} .
$$

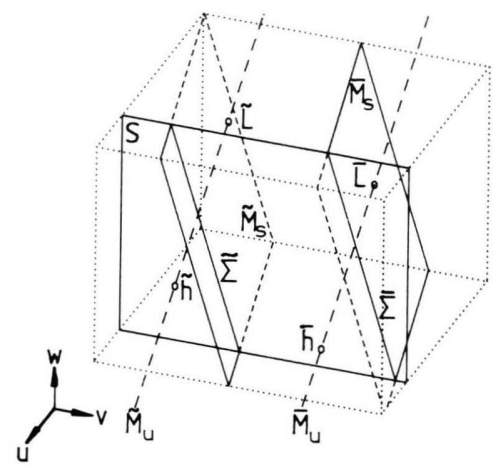

Fig. 1. Geometry of the phase space $T$ of the model system (3). The stable $\left(\hat{M}_{s}\right)$ and the unstable $\left(\hat{M}_{u}\right)$ manifolds of the two steady states $\hat{L}$ are shown. The virtual parts of the stable manifolds are dashed. The lines $\hat{\Sigma}$ and the points $\hat{h}$ are the intersections of the stable and unstable manifolds, respectively, with the separating plane $S$. Axes: $u=$ $-0.1 \ldots 0.2, v=-1.3 \ldots 0.5, w=-2 \ldots 1.4$. System parameters: $b=1, d=0.2, e=15, \mu=100, c=3.5$. 
These points will be called "homoclinoid" since $\hat{h}$ is the last entry point of a homoclinic trajectory (as will be shown shortly).

In analogy to the $\hat{\eta}_{\infty}$ above, we now define two

$$
\hat{x}_{0}:=\delta
$$

which give the $x$ coordinates of the two homoclinoid points $\hat{h}$.

The simple structure of both (44) and (48) is a consequene of the fact that no normalization of the eigenvectors $t^{i}$ was used (recall that the first row of the matrix $\mathbb{H}$ contains only l's, cf. [17]). For normalized $t^{i}$, we instead would obtain:

$$
\hat{x}_{0}=\delta / \mathbb{H}_{11}
$$

and

$$
\hat{\eta}_{\infty}=\delta / 2 \mathbb{H}_{12},
$$

respectively.

Within the stable and unstable manifolds, very special trajectories are partially located. When a solution spirals out from the real steady state $\bar{K}$ inside of $\bar{M}_{s}$ (the two-dimensional stable manifold), it crosses the separating plane $S$ in a point of $\bar{\Sigma}$, i.e., with vanishing $x$ component. The same trajectory may, after having crossed $S$ for an even number of times, eventually reenter $\bar{T}$ in such a way that its $y$ component is zero (no matter how unlikely this may be). In this singular situation, the system returns towards the steady state $\bar{K}$ inside the unstable manifold $\bar{M}_{u}$. It could leave this point again only after an infinite interval of the "time" $\varphi$. Such a trajectory is homoclinic in state space.

Since inside the unstable manifold $\bar{M}_{u}$ all phase information gets lost $(y=0)$, the homoclinic trajectory would leave the steady state - after the mentioned "infinite interval of time" - with an arbitrary phase. This means that a homoclinoid point $\hat{h}$ is mapped by a halfmap onto the whole line $\hat{\Sigma}$. Hence these two points are singular points of the two halfmaps.

Homoclinic trajectories in state space play an important role in the theory of excitable media (cf. [15]). They describe finite wavetrains on an infinite linear fiber [2], cf. also [10]. Homoclinic trajectories are also important in chaos theory (cf. [16]). A theorem of Shil'nikov [14] shows that under a mild condition mentioned above, there exist infinitly many periodic solutions of differing periods in a neighborhood of these trajectories.
A necessary condition for the appearance of a homoclinic solutions is

$$
\bar{h} \in S^{0-} .
$$

Only in this case is there the possibility of a return to $\bar{K}$. Equation (50) is satisfied in the parameter space of (3) for two intervals of the wavespeed $c$ which represent the stable and the unstable branch, respectively, of a certain dispersion relation $[2,17]$.

While the existence of a homoclinic trajectory is sufficient for chaos to exist, it certainly is not necessary. Instead of requiring a homoclinic trajectory (50), it suffices to consider systems fulfilling the following weaker axiom:

SY3: For all systems to be considered $\bar{h}$ is from $S^{-}$.

Here the superscript of $S$ (restricting it to the strip $S^{0}$ ) is missing. This axiom serves a purely technical purpose. It rules out systems which are not able to circle the unstable manifold $\bar{M}_{u}$ more than once before crossing the separating plane $S$. This sort of behavior is not of interest. Omitting these systems spares us having to distinguish different subcases in the following.

The above manifolds $\hat{M}_{s / u}$ are limiting cases of more general structures. All trajectories that differ in their initial conditions by a shift in phase only belong to the same, more general manifold.

As an example, let us look at solutions of (32) with initial condition $(\alpha, \beta)^{T}$. Then in cylindrical coordinates (with $r$ the absolute value, and $\chi$ the pase of $y$, respectively) the trajectory is found to be

$$
\begin{gathered}
x(\varphi)=\alpha e^{-\varrho \varphi}, \\
r(\varphi):=|y(\varphi)|=r_{0} e^{\varphi}, \\
\chi(\varphi):=\arg y(\varphi)=\chi_{0}+\omega \varphi,
\end{gathered}
$$

where $r_{0}=\mid \beta$, and $\chi_{0}=\arg \beta$. This shows that trajectories with initial conditions differing by a certain phase, continue to differ by exactly this phase through all values of $\varphi$.

Choosing $|\alpha|=\left|x_{0}\right|$ for convenience, we eliminate the parameter of representation $\varphi$ by using (51 a), arriving thereby at a purely geometrical representation of each manifold:

$$
\mathscr{M}\left(r_{0}\right):=\left\{\left.(x, y)^{T}|| y\left|=r_{0}\right| \frac{x}{\hat{x}_{0}}\right|^{-1 / o}\right\} .
$$

These manifolds may be called "dynamical" because the dynamical evolution of the system always 


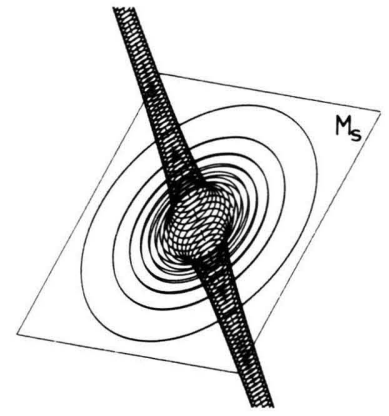

Fig. 2. Example of a dynamical manifold $\mathscr{M}\left(r_{0}\right)$ with $r_{0}=0.15$. The two-dimensional stable manifold $\left(M_{s}\right)$ of the steady state separates the two branches of the dynamical manifold (characterized by positive and negative values of $x$, respectively). The unstable manifold $\left(M_{u}\right)$ is the axis of rotational symmetry.

takes place on such a manifold as long as the system remains inside the same halfspace $\hat{T}$.

As (52) shows, each dynamical manifold preserves rotational symmetry, with radius $r$ depending on $x$ :

$$
r\left(x ; r_{0}\right)=r_{0}\left|\frac{x}{\hat{x}_{0}}\right|^{-1 / \varrho} .
$$

A dynamical manifold is thus characterized by a single real positive parameter, $r_{0}$ (see Figure 2). Hence a natural ordering on the dynamical manifolds is induced, this property will be used frequently.

\section{Intersection of the Dynamical Manifolds with the Plane $S$}

As all trajectories of each half system remain inside a dynamical manifold until they become eligible to switch dynamics (23), any entry and exit point of a trajectory must lie on the same intersection curve of the pertinent dynamical manifold with the separating plane $S$.

Before treating the general properties of the mentioned curves, let us have a brief look at the intersections of the $\mathscr{M}$ 's with another type of planes. The plaes $\hat{X}_{0}\left(x=\hat{x}_{0}\right)$ are parallel to the stable (twodimensional) manifolds of the steady states so that the intersection curves in question are circles. The great advantage of treating these planes is their having constant $x$ values.
We therefore define, in analogy to the halfmaps $\hat{P}$ (38), the two maps

$$
\bar{Q}: \bar{X}_{0} \rightarrow S^{+}, \quad \eta+\mathrm{i} \xi \mapsto \bar{Q}(\eta+\mathrm{i} \xi),
$$

and

$$
\tilde{Q}: \tilde{X}_{0} \rightarrow S^{-}, \quad \eta+\mathrm{i} \xi \mapsto \tilde{Q}(\eta+\mathrm{i} \xi) .
$$

The inverse of $\hat{Q}$ can be found using (51a). This equation yields, for the duration of evolution under the dynamics of each halfspace,

$$
\varphi_{\hat{Q}}=\frac{-1}{\varrho} \ln \left|\frac{x}{\hat{x}_{0}}\right| \text {. }
$$

Hence $\hat{Q}^{-1}$ can be calculated explicitly:

$$
\hat{Q}^{-1}(x, \xi)=\left(\frac{\delta-x}{2}+\mathrm{i} \xi\right) \exp \left(-(1+\mathrm{i} \omega) \varphi_{Q}\right) .
$$

All those structures for which the transformations (induced by the dynamics of the half systems) between $S^{+}$(or $S^{-}$, respectively) and $\hat{X}_{0}$ are nonsingular can be calculated inside the $\hat{X}_{0}$ planes. This offers an advantage because here the explicit map $\hat{Q}^{-1}$ is available.

Let us now look at the intersection of the dynamical manifolds with $S$. As the two partial dynamics are identical, it suffices to treat the halfmap $\bar{P}$ (the one possessing more structure due to the presence of a "real" steady state). We assume the convention $\bar{x}_{0}>0$ and suppress the bar where possible.

First a useful fact. The planes $X_{0}$ and $S$ intersect in a straight line parallel to $\Sigma$. On this line the real part of $y$ vanishes:

$$
\eta(x)=\eta\left(x_{0}\right)=\frac{\delta-x_{0}}{2}=0 .
$$

Therefore the imaginary part of $y$ (the numerical value of $\xi$ ) yields the pertinent $r_{0}$ of the dynamical manifold intersecting $S$ at that point.

In order now to investigate the intersection curve $\Gamma\left(r_{0}\right)$ of a dynamical manifold $\mathscr{M}\left(r_{0}\right)$ with $S$, we choose the geometrical $x$ representation given by (55). Let us first look at the $x$ interval, specific to a given manifold $/ /\left(r_{0}\right)$, within which an intersection occurs (Figure 3). The word "intersection" is used here in a more general, set theoretical sense (nontransversal points of contact belong to the intersection curve). Therefore the criterion for an intersection to exist is simply, whether at a given value of $x$, the dynamical manifold possesses both $u$ values that are greater or equal than $\delta$, and $u$ values less or 


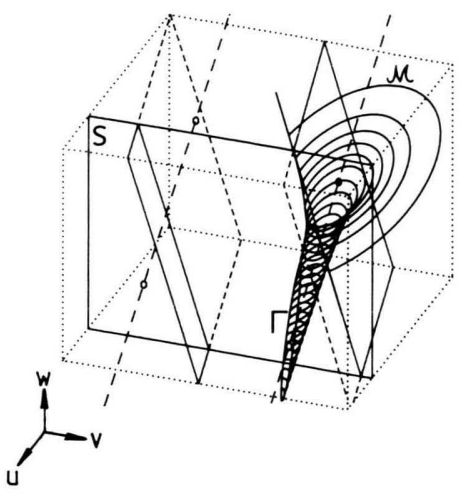

Fig. 3. Intersection of the separating plane $S$ with a dynamical manifold $\mathscr{M}\left(r_{0}\right)$, coming from the halfspace $\bar{T}$ with $r_{0}=0.15$. Axes and system parameters as in Figure 1.

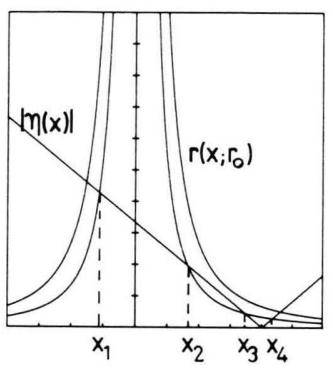

Fig. 4. The graph of the function $r\left(x ; r_{0}\right)$ shown for two different values of $r_{0}$. One sees that it intersects the piecewise-straight function $|\eta(x)|$ in either two or four points. The number of intersection points, in turn, determines the character of the intersection curve $\Gamma\left(r_{0}\right)$ (shown in Figure 5).

equal than $\delta$. It is sufficient to look at extremal values of $u$ for the $x$ in question. These extremal values are invariably reached at $\xi=0$ (no matter what the values of $x$ and $r_{0}$ ), because in this case the real part of $y$ becomes equal to the radius of the dynamical manifold (cf. (19)),

$$
\eta(x)= \pm|y(x)|= \pm r\left(x ; r_{0}\right) .
$$

Inserting (58) into (23), we obtain a condition for an intersection point to exist

$$
\eta(x) \mid<r\left(x ; r_{0}\right) .
$$

Here $r\left(x ; r_{0}\right)$ is the radius of the dynamical manifold (53), at the level $x$.

To find out in what $x$ interval the condition (59) can be fulfilled, we look for the zeros of the function $F\left(x ; r_{0}\right):=|\eta(x)|-r\left(x ; r_{0}\right)$ which is con- tained in (59). This function possesses one zero $\left(x_{1}\right)$ inside the interval $(-\infty, 0)$; either no zero or else two zeros $\left(x_{2}, x_{3}\right)$ (which may be degenerate) inside the open interval $\left(0, x_{0}\right)$; and exactly one zero $\left(x_{4}\right)$ inside $\left(x_{0}, \infty\right)$. This follows directly from Fig. 4 using some elementary algebraic reasoning [17].

Therefore, the intersection curves $\Gamma\left(r_{0}\right)$ of $\mathscr{M}\left(r_{0}\right)$ with $S$ are given by all points with

$$
r\left(x ; r_{0}\right)^{2}=\eta(x)^{2}+\xi^{2} .
$$

That is,

$$
\Gamma\left(r_{0}\right):=\left\{(x, \xi)^{T} \mid \xi=\xi(x)\right\},
$$

whereby

$$
\begin{aligned}
\xi(x) & = \pm \sqrt{r\left(x ; r_{0}\right)^{2}-\eta(x)^{2}} \\
& = \pm \sqrt{r_{0}^{2}\left|\frac{x}{x_{0}}\right|^{-2 / e}-\frac{(\delta-x)^{2}}{4}} .
\end{aligned}
$$

This was obtained by using the expressions (41) and (53) for $\eta(x)$ and $r\left(x ; r_{0}\right)$, respectively. Note that the $\Gamma$ 's, like the $\mathscr{M}$ 's, are characterized by one real parameter $r_{0}$ that induces an ordering on these curves. The $\Gamma$ curves can be classified according to the number of zeros of the function $F$. When there is just one zero $\left(x_{1}\right.$ or $\left.x_{4}\right)$ present inside $\mathbb{R}^{+}$ (or $\mathbb{R}^{-}$, respectively), the intersection curve is a simply connected curve that is shaped like a Greek letter $\Omega$. For three different zeros $\left(x_{2}, x_{3}, x_{4}\right)$ of $F$ present inside $\mathbb{R}^{+}$, the above function $\xi(x)$ is realvalued on the two disjoint intervals $\left(0, x_{2}\right]$ and $\left[x_{3}, x_{4}\right]$, meaning that the intersection curve $\Gamma\left(r_{0}\right)$ consists of a closed curve, called an isola, plus an open arc (called base line) that hugs $\Sigma$ for large values of $|\xi|$.

Note that the curves $\Gamma\left(r_{0}\right)$ of $(60)$ depend only on one of the two canonical parameters describing the sytem dynamics, namely $\varrho$, and not on the angular velocity $\omega$.

When the zeros $x_{2}$ and $x_{3}$ of the function $F$ are degenerate, the two branches of an $\Omega$-curve coalesce (or equivalently, the base line touches the isola) and form a Cartesian leaf. This limiting case is of special interest because this curve divides between the (as we shall see) rather simple behavior of the halfmap on an $\Omega$-curve and the (potentially very rich) behavior on an isola. Secondly, the point in which the two branches of the leaf meet can be calculated explicitly; cf. Figure 5. 

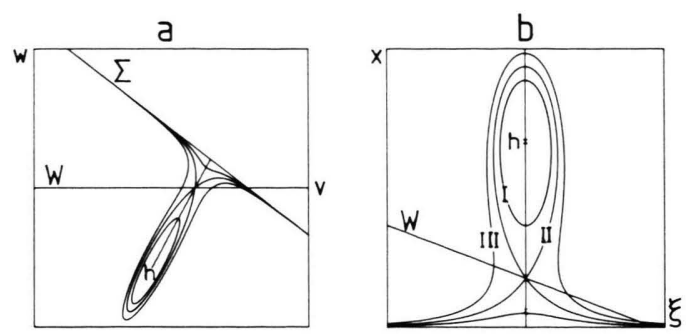

Fig. 5. Intersection curves $\Gamma\left(r_{0}\right)$, of one branch of a dynamical manifold $\mathbb{M}\left(r_{0}\right)$, with the separating plane $S$. The three possible types (see b) are: (I) an isola with its corresponding base line, (II) a Cartesian leaf (as a limiting case), and (III) an $\Omega$-curve. In part (a), these curves are visualized in the $S$-plane of the model system. In part (b), the same curves are shown in a model independent $x, \xi$ representation (in diagonalized coordinates). This representation will be used further on. Axes for (a): $v=$ $\bar{V}-0.3 \ldots \bar{V}+0.3, w=-3 \ldots 3 ;$ system parameters for (a): $b=1, d=0.2, e=15, \mu=100, c=3.5$. Axes for (b): $\xi=-0.5 \ldots 0.5, x=0 \ldots 1.7$; canonical system parameters for (b): $Q=5, \omega=2, \delta=1$.

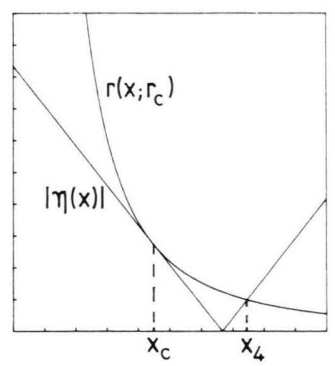

Fig. 6. Demonstration that, for $r_{0}=r_{c}$, the two graphs of $\eta(x)$ and $r\left(x ; r_{0}\right)$ meet tangentially at one point $\left(x_{c}\right)$ This is the criterion for determining both $r_{c}$ and $x_{c}$ (see text).

Let us first do this (Figure 6). If the zeros $x_{2}$ and $x_{3}$ coalesce for a certain $r_{0}=r_{c}$ at $x_{2}=x_{3}=x_{c}>0$, the two conditions

$$
r\left(x_{c} ; r_{c}\right)=\frac{\delta-x_{c}}{2}
$$

and

$$
\begin{aligned}
\frac{\mathrm{d}}{\mathrm{d} x} r\left(x_{c} ; r_{c}\right) & =\frac{-1}{\varrho x_{c}} r\left(x_{c} ; r_{c}\right) \\
& =-\frac{1}{2}=\frac{\mathrm{d}}{\mathrm{d} x} \eta(x)
\end{aligned}
$$

have to be fulfilled simultaneously. ( $x_{c}$ is a zero point of $F$ and the slopes of $\eta\left(x_{c}\right) \mid$ and $r\left(x_{c} ; r_{c}\right)$ have to be equal.) The second equation can be solved for $x_{c}$ :

$$
\begin{aligned}
2 r_{c}\left|\frac{x_{c}}{x_{0}}\right|^{-1 / \varrho} & =2 r_{c}\left(\frac{x_{c}}{x_{0}}\right)^{-1 / \varrho}=\varrho x_{c} \\
x_{c} & =\left(\frac{2 r_{c}}{\varrho}\right)^{\varrho /(\varrho+1)} x_{0}^{1 /(\varrho+1)} .
\end{aligned}
$$

Inserting this result into (61) yields

$$
\begin{aligned}
& 2 r_{c}\left(\frac{2 r_{c}}{\varrho}\right)^{-1 /(\varrho+1)} x_{0}^{1 /(\varrho+1)}=\delta-\left(\frac{2 r_{c}}{\varrho}\right)^{\varrho /(\varrho+1)} x_{0}^{1 /(\varrho+1)} \\
& 2 r_{c}=\delta^{(\varrho+1) / \varrho} x_{0}^{-1 / \varrho}(\varrho+1)^{-(\varrho+1) / \varrho} .
\end{aligned}
$$

Making use of the identity $\delta=x_{0}=2 \eta_{\infty}$, we obtain

$$
r_{c}=\eta_{\infty}(\varrho+1)^{-(\varrho+1) / \varrho} .
$$

When now inserting (64) into (63), we finally arrive at the $x$ component of the "Cartesian point",

$$
x_{c}=\frac{x_{0}}{\varrho+1} .
$$

The corresponding $\xi$ component of this point is zero by construction. Therefore the $x$ component alone may be called "Cartesian point" for short. This point possesses a second interesting feature: It is situated on the boundary between range and domain of the halfmap $\bar{P}$, i.e., on the line $W$. This follows from a straightforward calculation using (19) [17].

The radius $r_{c}$ characterizing the Cartesian leaf is critical in the sence that for $r_{0}<r_{c}$, the $\Gamma\left(r_{0}\right)$ are isolae plus base lines, while for $r_{0}>r_{c}$ the intersection curves are $\Omega$-curves (cf. [17]).

\section{The Case of Complicated Isolae}

The examples of intersection curves $\Gamma$ presented so far seem to suggest that all isolae are situated completely inside $S^{-}$, the domain of the halfmap $\bar{P}$. In fact, this is the simplest and most common case, but it is not the only possible one. Any isola that is situated completely inside $S^{-}$can be called simple since it is mapped by $\bar{P}$ on a partition of its corresponding base line. Some isolae, however, may cross $W$. They will be called complicated isolae (Figure 7). In the latter case the isola is partially mapped to itself and partially to the base line. 
a
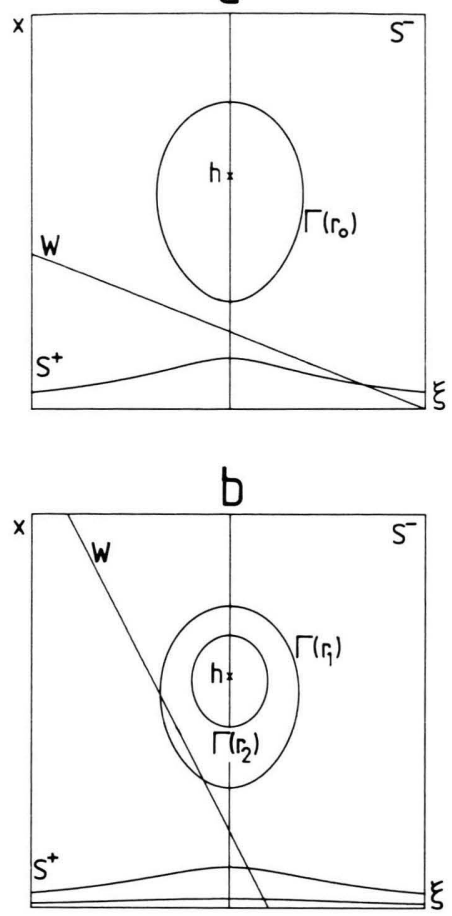

Fig. 7. Occurrence of simple and complicated isolae. In part (a), the canonical parameters are chosen so that the system can only possess simple isolae. Parameters: $\varrho=2$, $\omega=1, \delta=1, r_{0}=0.95 r_{c}$. In part (b), it is demonstrated that for values of $r_{0}$ close to $r_{c}$, a complicated isola $\left(\Gamma\left(r_{1}\right)\right)$ appears, while nevertheless for small enough values of $r_{0}$, the isolae are still of the simple type $\left(\Gamma\left(r_{2}\right)\right)$. Parameters: $\varrho=2, \omega=5, \delta=1, r_{1}=0.9 r_{c}, r_{2}=0.5 r_{c}$. Axes for both pictures: $\xi=-0.5 \ldots 0.5, x=0 \ldots 1.7$.

Here a discontinuity appears in the halfmap $\bar{P}$ since the image of the connected portion of the isola, situated in $S^{-}$, becomes disconnected.

So far, we have looked at features (described by $r_{0}$, for example) within a single system. Now let us look at different systems - i.e., at different points of the canonical parameter space - to see inside which regions of that space complicated isolae appear. We find:

Theorem: For all systems fulfilling the condition

$$
\omega^{2}<\varrho+1
$$

all isolae are simple, that is, are completely from $S^{-}$.

For a proof see the Appendix.

As a simple consequence of the proof (see Appendix) we formulate the following
Corollary: In all systems fulfilling

$$
\omega^{2}>\varrho+1
$$

complicated isolae appear.

The appearance of complicated isolae may be visualized in the limiting "low" and "high" frequency cases, respectively. In the first case (where $\left.\omega^{2} \ll Q+1\right)$, the relaxation of the system in the direction of the real eigenvetor is much faster than the focal motion, so a trajectory with an entry point situated on an isola will exit $\bar{T}$ close to $\Sigma$. That means the exit point is situated on the base line. For the oher limiting case (where $\omega^{2} \gg \varrho+1$ ), the motion inside $\bar{T}$ is mainly governed by the focal part of the dynamics. So the exit point shows almost the same $x$ value as the entry point and thus is situated on the isola. For intermediate $\omega, \varrho$ ratios, both complicated and simple isolae may coexist, depending on their $r_{0}$ values.

This theorem and its corollary allow us to distinguish between those systems that possess complicated isolae and those that do not. By now all possible types of intersection curves $\Gamma\left(r_{0}\right)$ have been considered. These results permit a qualitative picture of the action of the halfmap.

\section{Action of a Halfmap on the Intersection Curves}

The two halfmaps induced by the partial dynamics in the halfspaces $\bar{T}$ and $\tilde{T}$ are of the same type because their dynamics differ only by the nonhomogeneous part on the right-hand side of (3). One of them $(\bar{P})$ shows more structure by virtue of the real steady state being present. Since the second dynamics is governed by a virtual steady state, the only $\Gamma$ 's possible are $\Omega$-curves. So the halfmap $\tilde{P}$ acts more or less like a reflection along the straight line $W$ (cf. [17]). Hence it suffices to discuss only the first halfmap, $\bar{P}$.

As our halfmap can be formulated only implicitly, three general strategies for investigating it are open in principle. The first approach consists in solving (40a) numerically. This was done in [18]. The second method is based on the explicit map $\bar{Q}^{-1}$ described in $(54-56)$. This map like $\bar{P}$ is induced by the dynamics from the halfspace $\bar{T}$, but without any restriction analogous to $(40 \mathrm{~b})$ occurring. That is, it is constructed as if there were just one 
dynamics, acting on the whole state space, present. All possible intersection points of a trajectory with the separating plane $S$ are mapped by $\bar{Q}^{-1}$ onto the same point (in $\bar{X}_{0}$ ). Hereby the "mapping times" $\varphi_{\hat{Q}}$ are different. Hence no global one-to-one correspondence between the points of $S^{-}$(the domain of $\bar{P}$ ) and those of $\bar{X}_{0}$ exists. Nevertheless, such a correspondence can be obtained by introducing a specific selection rule (for the $\varphi_{\hat{Q}}$ ), permitting some additional results to be found [19].

The third approach is the one to be discussed here. It makes use of the intersection curves of the dynamical manifolds $\mathscr{M}\left(r_{0}\right)$ with the separating plane $S$. These curves are known explicitly (60). The image $\left(p^{\prime}\right)$ of any point $p$ from $S^{-}$is located inside $S^{+}$on the same intersection curve $\Gamma\left(r_{0}\right)$ as $p$ itself. The different ways in which the halfmap $\bar{P}$ can act on each of the three types of intersection curves can be put together to obtain a coherent picture of the structure of the halfmap.

As a general property of all halfmaps we find that the $x$ component of the image of a point $p$ is always less than $p_{x}$, the $x$ component of $p$ itself. Hence a segment of a base line is always mapped onto another segment of the same base line. Also the image of a (simple) isola is always located on the corresponding base line.

More specifically, it turns out that for the majority of the $\Omega$-curves (all those possessing only one intersection point with the straight line $W$ ), the halfmap has a very simple action. The trajectory, generating the map, goes around the unstable manifold for only (approximately) one half turn. The halfmap therefore acts as a kind of reflection along the straight line $W$ (in analogy to what is the case in $\tilde{T}$ ).

For $\omega^{2}<\varrho+1$, however, there exist also $\Omega$-curves (for $r_{0}$ close to $r_{c}$ ) that intersect $W$ in three points. In this case, the arc $a b$ (cf. Fig. 8) of the $\Omega$-curve located inside $S^{-}$is cut into two parts at a point $s$. These two portions are mapped onto two different segments of the portion of the $\Omega$-curve which lies inside $S^{+}$. Figure 8 shows that the arc as is mapped onto $a s^{\prime}$ whereas $b c$ is the image of $b s$.

More precisely it can be shown that $\Omega$-curves are cut open whenever their $r_{0}$ is less than some critical $r_{0}$. The value of this threshold is

$$
r_{w}=\eta_{\infty} \frac{\varrho}{\omega}\left|\frac{x_{w}}{x_{0}}\right|^{(\varrho+2) / 2 \varrho},
$$

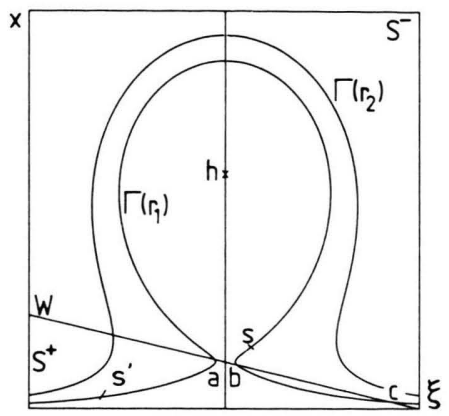

Fig. 8. Demonstration of two facts: (i) The $\Omega$-curve $\Gamma\left(r_{2}\right)$ possesses but one intersection point with $W$. The arc inside $S^{-}$is mapped homeomorphically into that inside $S^{+}$ ("mirrored" across $W$ ). (ii) The $\Omega$-curve $\Gamma\left(r_{1}\right)$ intersects $W$ in the three points $a, b$, and $c$. It is thus "cut open" so that the image of the arc as goes to the left in $S^{+}$while the image of $s b$ goes to the right (onto $c b$ ). Axes: $\xi=-0.5 \ldots 0.5, x=0 \ldots 1$.7. Parameters: $\varrho=4, \omega=1, \delta=1$, $r_{1}=1.002 r_{c}, r_{2}=1.25 r_{c}$. (Note that both here and in the following two Figures, the image points that were indicated by a prime are shown only schematically, since the true image points in this case are located far beyond the boundaries of the plotting area.)

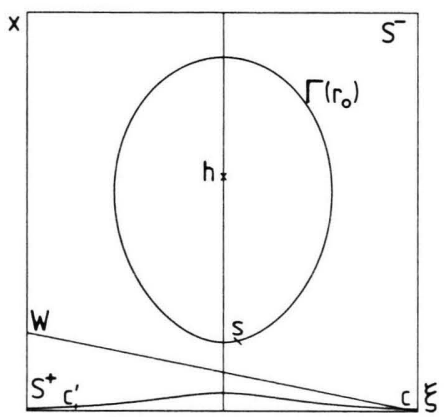

Fig. 9. A simple isola is cut open in the point $s$ and mapped onto the open arc $c c^{\prime}$ in the base line. Axes: $\xi=-0.5 \ldots 0.5, x=0 \ldots 1.7$. Parameters: $\varrho=5, \omega=1$, $\delta=1, r_{0}=0.95 r_{c}$.

where

$$
x_{w}=x_{0} \frac{\omega^{2}+1}{(\varrho+1)^{2}+\omega^{2}}
$$

(cf. [19]).

As to isolae, the halfmap may show several types of behavior again. Every simple isola (a closed curve) is mapped onto an open segment of the corresponding base line, so there is always a point $s$ on the isola where it is cut open (Figure 9). The images of neighboring points (to the left and the right) of $s$ are always separated by the halfmap $\bar{P}$. This separation of nearby points under the action of a halfmap 
may give rise to a positive "Lyapunov exponent" [20] of the overall Poincaré map $P$ [10]. This is a first chaos-generating mechanism (cf. also [19]).

For complicated isolae, the situation is more involved (Figure 10). This type of isolae exists whenever $\omega^{2}>\varrho+1$ (see (66)). In this case, $r_{w}$ is less than $r_{c}$ (see (67)) and all isolae characterized by a radius $r_{0}$ from the interval $\left(r_{w}, r_{c}\right)$ are complicated [19].

For a complicated isola only the segment inside $S^{-}$is subjected to the map $\bar{P}$. This portion is mapped partially onto the other part of the isola (inside $S^{+}$) and partially into the base line. While in the case of simple isolae, the image of a planar region inside $S^{-}$(say the interior of a isola) was stretched and cut open but remained simply connected, in the present case the planar region bounded by $W$ and $\Gamma\left(r_{0}\right) \cap S^{-}$is mapped onto two disjoint regions of $S^{+}$. Here connectivity is lost. This is a second separation-generating (and hence chaosproducing) mechanism found in halfmaps.

A third mechanism involving the "travelling times" $\tau$ (solutions of (40a)) comes into play at even larger values of the ratio $\omega / \varrho$, see [19].

The main effect is the same in all three cases. A portion of an intersection curve $\Gamma\left(r_{0}\right)$ situated in $S^{-}$is "cut in two" and the images of nearby points to the left and the right of the cutting point are separated. The various mechanisms of separation differ in the number of "cutting open points" present on an intersection curve. The geometric locus of all "cutting open points" for different $\Gamma\left(r_{0}\right)$ can be calculated analytically [19].

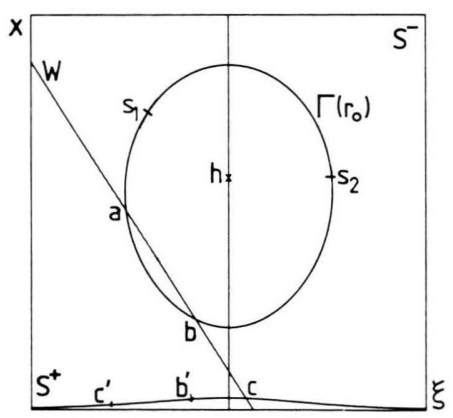

Fig. 10. Mapping situation of a complicated isola. The arc $a s_{1}$ is "mirrored", becoming $a b$, while the rest of the isola $\left(s_{1} b\right)$ is again cut open in the point $s_{2}$ (cf. Fig. 9). Note the reversal of mutual orientation between the images of the arcs $s_{1} s_{2}$ (becoming $b^{\prime} c^{\prime}$ ) and $s_{2} b$ (becoming $c b^{\prime}$ ). Axes: $\xi=-0.5 \ldots 0.5, x=0 \ldots 1.7$. Parameters: $\varrho=5, \omega=8$, $\delta=1, r_{0}=0.9 r_{c}$.
The discussed cutting open mechanisms occur in addition to a stretching in $\xi$ direction and a shrinking in $x$ direction, due to the saddle focus behavior of the steady state.

\section{Discussion}

The model system discussed here is a well known nerve conduction equation frequently treated in the literature under different aspects. To the best of our knowledge, the technique of Poincaré halfmaps has not been applied to this class of problems before. The theory of halfmaps induced by three-dimensional linear flows is yet to be worked out fully. In the present paper, we attempted to describe the case when the linear flow is of saddle-focus type.

Poincare halfmaps, and the intersection curves of dynamical manifolds with the separating plane $S$, were studied numerically in $[7,18,21]$. Here the intersection curves are explicitly constructed in an analytical fashion for the first time. Thereby the canonical parameter space could be characterized analytically to some extent. The main pertinent result is that the curve $\omega^{2}=\varrho+1$ in the $\varrho, \omega$ plane of the five-dimensional canonical parameter space acts as a double boundary. It is a limiting curve (as far as the actions of the halfmap is concerned) both for isolae and for $\Omega$-curves.

Below that curve, only simple isolae are possible. Hence the only possibility for a chaotic solution to appear in this case is by means of the first "cutting open mechanism" of separation. This mechanism acts on all isolae as well as on some of the $\Omega$-curves (characterized by an $r_{0}$ close to $r_{c}$ ).

Above the same curve, $\Omega$-curves that are cut open by the halfmap disappear but at the same time complicated isolae appear. The first cutting open mechanism (acting on all isolae) is still present. In addition, however, every complicated isola has a disconnected image. Thus a second separating mechanism (leading to a second cutting open point on a complicated isola) comes into play. Both cutting open mechanisms can give rise to chaotic solutions of the system.

The other main result of the present paper is a proof for the appearance of chaotic solutions in the neighborhood of homoclinic trajectories in state space, for the present class of systems, provided $\varrho>1$. This follows from the fact that Shil'nilov's 
theorem [14] is applicable (see Section 5). A homoclinic trajectory (if it exists in conjunction with the second halfmap) necessarily passes through the homoclinoid point $\bar{h}$. The "simple cutting open mechanism" (the only one present for arbitrarily small values of $r_{0}$, as is the case in a neighborhood of the homoclinoid point $\bar{h}$ ) is thus responsible for generating a countable number of Smale [22] horseshoes (cf. [23]). This means that even the simplest possible separating structure already supports an infinitly rich dynamical behavior in the present class of systems.

The two example sets of parameter values presented in [10] visualize the appearance of complicated isolae and the corresponding separation mechanism. Although in both cases the condition $\omega^{2}>\varrho+1$ is fulfilled, only the first of the two chaotic attractors (the one with $c=3.5$ ), also makes actual use of the second separating mechanism (leading to disconnected images of complicated isolae). In the second case $(c=9)$, only a neighborhood of a homoclinic orbit in state space is visited by the trajectory running inside the attractor, i.e., only a vicinity of the homoclinoid point $\bar{h}$ is made use of by the overall Poincare map. This explains the rather simple structure of this second attractor so that it could be described rather well in terms of a onedimensional map.

To sum up, a Poincaré halfmap induced by a linear flow of saddle-focus type has the capability of separating arbitrarily close points and thus may lead to chaotic solutions of the system. While on some of the $\Omega$-curves only the simplest cutting open mechanism occurs, on isolae three different types are possible.

It goes without saying that the "potentially chaosproducing" mechanisms, described above for a single halfmap $\bar{P}$, of course only become manifestly chaos-producing if the second halfmap $\tilde{P}$ does not precisely undo the separation of nearby points (which is infinitly unlikely), and if, moreover, the regions inside $S^{-}$in which the separations appear are mapped back to themselves by the second halfmap. Some examples where this occurs in piecewise-linear continuous systems were discussed numerically by several authors [10, 18, 24]. An analytical negative criterion (stating that no chaotic solutions can occur if the intersection of the "separating region" with its image under the whole Poincaré map $P$ is empty) has also been found [25].
To conclude, the occurrence of chaos in a piecewise-linear equation for a one-dimensional excitable medium can be explained for a whole class of systems by means of analytically described separating mechanisms.

\section{Appendix}

Let us first point to two general properties of intersection curves.

(1) If $r_{1}<r_{2}$, then the intersection curve $\Gamma\left(r_{1}\right)$ lies completely inside the region of $S$ bounded by $\Gamma\left(r_{2}\right)$ and $\Sigma$.

(2) Every isola is the boundary of a convex region in $S$.

For the proofs see [17].

Proof of the Theorem (Section 7). All isolae are situated inside the Cartesian leaf, thus it will suffice to show that this curve does not intersect $W$ for values of $x$ greater than $x_{c}$ if $\omega^{2}<\varrho+1$.

As the Cartesian point $x_{c}$ is situated on $W$, by convexity there cannot occur any further intersection point for $x$ values greater than $x_{c}$ if the slope of the relevant branch of $\Gamma\left(r_{c}\right)$ at this point is less or equal to zero (in a $v, w$ representation of $S$ ). It will be shown specifically that for $\omega^{2}=\varrho+1$ the Cartesian leaf is tangential to (uniquely touches) $W$ in the Cartesian point $x_{c}$.

On the Cartesian leaf $\Gamma\left(r_{c}\right)$, we obtain for the relevant branch (the one with negative $\xi$ values) the analytical expression for $\xi(x)$ :

$$
\xi(x)=-\sqrt{r\left(x ; r_{c}\right)^{2}-\eta(x)^{2}}
$$

with

$$
r\left(x ; r_{c}\right)=r_{c}\left|\frac{x}{x_{0}}\right|^{-1 / \varrho}
$$

and

$$
\eta(x)=(\delta-x) / 2,
$$

as was found earlier (60). This yields for the $w$ component of the points of this branch of the Cartesian leaf:

$w(x)=-\varrho x+2 \operatorname{Re}[(1+\mathrm{i} \omega)(\eta(x)+\mathrm{i} \xi(x))]$.

We have to show that the slope of $w(x)$ vanishes at $x_{c}$ for $\omega^{2}=\varrho+1$. Taking the derivative of $w(x)$ 
with respect to $x$ we obtain

$$
\begin{aligned}
\frac{\mathrm{d}}{\mathrm{d} x} w(x) & =-\varrho+2\left(\frac{\mathrm{d}}{\mathrm{d} x} \eta(x)-\omega \frac{\mathrm{d}}{\mathrm{d} x} \xi(x)\right) \\
& =-\varrho-1+2 w \frac{2 r\left(x ; r_{\mathrm{c}}\right) r^{\prime}\left(x ; r_{\mathrm{c}}\right)+\eta(x)}{2 \xi(x)} .
\end{aligned}
$$

Inserting this into (A.3) yields

$$
\begin{aligned}
\frac{\mathrm{d}}{\mathrm{d} x} w\left(x_{c}\right) & =-(\varrho+1)+\omega \sqrt{\varrho+1} \\
& =-(\varrho+1)\left(1-\frac{\omega}{\sqrt{\varrho+1}}\right) .
\end{aligned}
$$

This leads for $x=x_{\mathrm{c}}$ to an undefined expression (of the form " $0 / 0$ ") in the third term. It is convenient to investigate the behavior of $\left(\xi^{\prime}\right)^{2}$ rather than that of $\xi^{\prime}$ :

$$
\begin{aligned}
\lim _{x \rightarrow x_{c}} \xi^{\prime}(x)^{2} & =\frac{\lim _{x \rightarrow x_{c}} \frac{\mathrm{d}}{\mathrm{d} x}\left[r\left(x ; r_{c}\right) r^{\prime}\left(x ; r_{c}\right)+\frac{1}{2} \eta(x)\right]}{\lim _{x \rightarrow x_{c}} \frac{\mathrm{d}}{\mathrm{d} x}\left[r\left(x ; r_{\mathrm{c}}\right)^{2}-\eta(x)^{2}\right]} \\
& =\lim _{x \rightarrow x_{c}} \frac{r\left(x ; r_{c}\right)^{2}}{\varrho^{2} x^{2}}(\varrho+2)-\frac{1}{4} \\
& =\frac{(\varrho+1)}{4} .
\end{aligned}
$$

Therefore for $\omega^{2}<\varrho+1$ the derivative of $w(x)$ is negative at $x_{c}$. Hence the relavant branch of the Cartesian leaf $\Gamma\left(r_{c}\right)$ and thus all isolae contained inside this leaf are situated completely inside of $S^{-}$.

Q.E.D.
[1] R. Rosen, Dynamical System Theory in Biology, Wiley-Interscience, New York 1970.

[2] C. Kahlert and O. E. Rössler, Z. Naturforsch. 38a, 648 (1983).

[3] J. Rinzel and J. B. Keller, Biophys. J. 13, 1313 (1973).

[4] C. T. Sparrow, J. Math. Analysis and Appl. 83, 275 (1981).

[5] C. Kahlert, Existence and uniqueness of solutions of piecewise-defined dynamical systems, submitted to SIAM J. Math. Analysis, 1985.

[6] H. Poincaré, Les Methodes Nouvelles de la Mechanique Celeste, Gauthier-Villas, Paris 1892.

[7] B. Uehleke, Chaos in a piecewise-linear system: analytical results (in German), Ph.D. Thesis, University of Tübingen, 1982; B. Uehleke and O. E. Rössler, Z. Naturforsch. 38a, 1107 (1983).

[8] J. W. Evans, N. Fenichel, and J. A. Feroe, SIAM J. Appl. Math. 42, 219 (1982).

[9] G. A. Carpenter, SIAM J. Appl. Math. 36, 334 (1979).

[10] C. Kahlert and O. E. Rössler, Z. Naturforsch. 39a, 1200 (1984).

[11] J. E. Dayhoff, Bull. Math. Biol. 46, 529 (1984).

[12] K. Kirby and M. Conrad, Bull. Math. Biol. 46, 765 (1984).

[13] C. Kahlert, Dispersion relations for a class of reaction-diffusion-equations (in German), Diploma Thesis, University of Tübingen, 1981.

[14] L. P. Shil'nikov, Sov. Math. Docl. 6, 163 (1965).
[15] G. A. Carpenter, J. Diff. Eq. 23, 335 (1977).

[16] P. Glendinning and C. Sparrow, Local and global behaviour near homoclinic orbits, Preprint 1983.

[17] C. Kahlert, Infinite nonperiodic wavetrains in a class of reaction-diffusion-equations - analytical properties of Poincaré halfmaps (in German), Ph.D. Thesis, University of Tübingen, 1984.

[18] B. Uehleke and O. E. Rössler, Z. Naturforsch. 39a, 342 (1984).

[19] C. Kahlert, Analytical properties of the chaos-producing mechanisms in piecewise-linear dynamical systems, in preparation.

[20] D. Ruelle, Sensitive dependence on initial conditions and turbulent behavior of dynamical systems, in $\mathrm{Bi}$ furcation Theory and its Applications in Scientific Disciplines, O. Gurel and O. E. Rössler, Editors, Ann. New York Acad. Sci. 316, 408 (1979).

[21] O. E. Rössler, The gluing-together principle and chaos, in Nonlinear Problems of Analysis in Geometry and Mechanics, M. Atteia, D. Bancel, and I. Gumowski, Editors, Pitman, Boston 1981, pp. 50-56.

[22] S. Smale, Bull. Amer. Math. Soc. 73, 747 (1967).

[23] L. P. Shil'nikov, Math. USSR Sbornik 10, 91 (1970).

[24] T. Matsumoto, L. O. Chua, and M. Komuro, A chaotic Attractor from Chua's circuit, IEEE Trans. CAS, Aug. 1985, in press.

[25] C. Kahlert, The composition of Poincare halfmaps and chaotic attractors, in preparation. 